\title{
Inadequate Quality of Surveillance after Curative Surgery for Colon Cancer
}

\author{
Carsten T. Viehl, $\mathrm{MD}^{1}$, Alex Ochsner, $\mathrm{MD}^{1,2}$, Urs von Holzen, $\mathrm{MD}^{2}$, Ramona Cecini, $\mathrm{MD}^{3}$, Igor Langer, $\mathrm{MD}^{1,4}$, \\ Ulrich Guller, MD, MHS ${ }^{1,5}$, Urban Laffer, $\mathrm{MD}^{3}$, Daniel Oertli, $\mathrm{MD}^{1}$, and Markus Zuber, $\mathrm{MD}^{2}$ \\ ${ }^{1}$ Department of Surgery, University Hospital Basel, Basel, Switzerland; ${ }^{2}$ Department of Surgery, Kantonsspital Olten, \\ Olten, Switzerland; ${ }^{3}$ Department of Surgery, Spitalzentrum Biel, Biel, Switzerland; ${ }^{4}$ Department of Surgery, Kantonsspital \\ Bruderholz, Bruderholz, Switzerland; ${ }^{5}$ Department of Surgery, Division of Visceral Surgery and Transplantation, \\ University Hospital Bern, Bern, Switzerland
}

\begin{abstract}
Background. Colon cancer patients are at risk for recurrence. Recurrent disease might be curable if detected early by surveillance. However, data on the quality of surveillance are scarce. The objective of this study is to analyze the quality of surveillance after curative surgery for colon cancer among a cohort of Swiss patients.

Patients and Methods. After curative surgery, 129 stage I-III colon cancer patients were followed by chart review, questionnaires, and phone interviews. National surveillance guidelines mandate periodic measurement of carcinoembryonic antigen (CEA) levels, abdominal ultrasound or computed tomography (US/CT), and colonoscopy. However, surveillance was left to the discretion of the treating physicians. Actual surveillance was compared with the recommendations in the guidelines.

Results. Datasets of all 129 patients were available. Median follow-up was 33.5 months (range 5.6-74.7 months). Eighteen patients (14.0\%) recurred during follow-up. Threeyear overall and disease-free survival were $94.7 \%$ and $83.5 \%$, respectively. Periodic CEA measurements, US/CT, and colonoscopies as recommended by the guidelines were performed in $32.8 \%, 31.7 \%$, and $23.8 \%$ of patients,
\end{abstract}

Carsten T. Viehl and Alex Ochsner equally contributed to this paper

Presented in part at the 62nd Annual Cancer Symposium of the Society of Surgical Oncology, Phoenix, AZ, March 5-8, 2009

(C) Society of Surgical Oncology 2010

First Received: 4 January 2010;

Published Online: 29 April 2010

M. Zuber, MD

e-mail: markus.zuber@spital.so.ch respectively. Forty-four patients $(34.1 \%)$ received adjuvant chemotherapy. For these patients there was a trend towards better compliance with national surveillance guidelines than for patients without adjuvant chemotherapy.

Conclusions. The quality of surveillance after curative surgery for colon cancer among a cohort of Swiss patients is inadequate. Further education of health care professionals and patients regarding the potential life-saving benefits of surveillance is imperative. It is cardinal that quality of surveillance is critically analyzed in other countries with different health care systems as well.

Colon cancer is a common tumor in the Western world. Despite new therapeutic regimens, mortality rates remain considerable. In the USA, about 50,000 deaths from colon cancer are anticipated annually. ${ }^{1,2}$ Patients can be cured if the cancer is detected early and completely resected. However, up to $30-44 \%$ of colon cancer patients will develop local recurrences and/or distant metastases even after curative surgery. ${ }^{3-5}$ In the majority of cases, the cancer will recur within the first 2-3 years after resection of the primary tumor. ${ }^{3,5,6}$ A high recurrence rate and the known early advent of recurrences are the rational for surveillance after curative surgery for colon cancer. ${ }^{3-5}$ There is compelling evidence from three meta-analyses that patients benefit from intensive surveillance after curative surgery for colon cancer and that mortality can be significantly reduced by surveillance..$^{7-9}$ Additional evidence from large-scale, adequately powered trials that are currently underway is eagerly awaited. ${ }^{10,11}$

Surveillance aims to detect local tumor recurrence, metastases, and metachronous colorectal cancer in an early stage when treatment might still be curative. ${ }^{12-14} \mathrm{~A}$ prerequisite for meaningful surveillance is that the patient 
qualifies for potential surgical treatment of cancer recurrence regarding age and comorbidities. Actual multimodal therapeutic regimens show good results for curative treatment of colorectal cancer recurrence. Five-year survival rates of up to $58 \%$ after curative resection of hepatic metastases can be achieved. $^{15-17}$ Moreover, modern neoadjuvant chemotherapy regimens (e.g. FOLFOX or FOLFIRI) in addition with biologic agents (e.g., bevacizumab, cetuximab) successfully downstage resectable liver metastases; consequently, more $\mathrm{R} 0$ resections and a 5-year survival rate of up to $50 \%$ can be achieved. $^{18,19}$ Therefore nihilism regarding treatment of colorectal cancer recurrence is no longer justified, and surveillance programs become increasingly important.

Surveillance programs have therefore been recommended for colorectal cancer patients in several countries, and appropriate surveillance guidelines have been issued by various gastroenterological, oncological, and/or surgical societies. $^{12-14}$ The Swiss Society of Gastroenterology (SGG), for example, regularly publishes the current Swiss recommendations for surveillance after curative resection for colorectal cancer. The SGG elaborated recommendations for the first time in 1996. Updated recommendations followed in 1999, 2003, and 2007. ${ }^{12,20,21}$

Despite the tremendous importance of surveillance after curative resection of colon cancer, there is very little data on adherence to surveillance guidelines in the scientific literature. Hence, the quality of surveillance after curative surgery for colon cancer is largely unknown. Therefore, the objective of this study was to analyze the quality of surveillance after curative surgery for colon cancer among a cohort of Swiss patients.

\section{PATIENTS AND METHODS}

Data presented in this study are based on follow-up of 165 patients with resected colon cancer who were enrolled into the prospective "Swiss Multicenter Trial Sentinel Lymph Node Procedure in Colon Cancer" (NCT 00826579) between April 2000 and December $2005 .^{22}$ The study was conducted at three Swiss academic and university-affiliated institutions in different geographic regions of Switzerland. Follow-up of the patients was not part of the initial study protocol. All treating physicians received a discharge letter where surveillance according to national guidelines was recommended to the treating physicians. However, actual surveillance was left to the discretion of the respective treating physicians: surveillance for patients not receiving adjuvant chemotherapy was organized by their respective general practitioners; surveillance for patients with adjuvant chemotherapy was initiated by the medical oncologists, and after completion of chemotherapy managed by general practitioners.
Patients with primary metastatic disease [American Joint Committee on Cancer (AJCC) ${ }^{23}$ stage IV; $n=18$ ], patients who deceased within 30 days after surgery $(n=6)$, patients refusing surveillance $(n=1)$, and patients who did not qualify for surveillance due to age and severe comorbidity ( $n=11)$ were excluded, leaving 129 eligible patients for analysis.

All pertinent in- and outpatient files were retrospectively reviewed, and the treating general practitioners, gastroenterologists, and patients received a questionnaire. In addition, all patients still alive were contacted by phone at the time of follow-up. We compared the actual surveillance, which had been performed during follow-up, with the national surveillance guidelines (Tables 1 and 2). As the surveillance guidelines changed during the study period, the analysis of expected surveillance compared with the actual surveillance was adjusted for the Swiss guidelines at the time of surveillance. Parameters analyzed were: measurements of carcinoembryonic antigen (CEA); diagnostic imaging: ultrasound (US) or computed tomography (CT); and colonoscopy. Although CT scans were not officially recommended until 2007, abdominal CT scan was considered a valid substitute for ultrasound. Surveillance according to Swiss guidelines was defined as an examination conducted within \pm 2 months for CEA and US/CT, and within \pm 4 months for colonoscopy, respectively, from the time point recommended in the guidelines. At each time point, e.g., 6, 12, or 18 months postoperatively, the percentages of patients receiving surveillance according to the guidelines were calculated. The results are shown as the median percentage and range of surveillance according to the guidelines for each recommended examination. In case

TABLE 1 National surveillance guidelines for colon cancer 1999 as published by the Swiss Society of Gastroenterology ${ }^{21}$

\begin{tabular}{lccccccc}
\hline Months postoperatively & 6 & 12 & 18 & 24 & 36 & 48 & 60 \\
\hline Ultrasound & & + & & + & + & & \\
Colonoscopy & & & & & & & + \\
Physical examination, CEA, CT scan & No recommendation & &
\end{tabular}

Physical examination, CEA, CT scan No recommendation

TABLE 2 National surveillance guidelines for colon cancer 2003 as published by the Swiss Society of Gastroenterology ${ }^{20}$

\begin{tabular}{lccccccc}
\hline Months postoperatively & 6 & 12 & 18 & 24 & 36 & 48 & 60 \\
\hline Physical examination & + & + & + & + & + & + & + \\
CEA measurement & + & + & + & + & + & + & + \\
Ultrasound & & + & & + & + & + & + \\
Colonoscopy & \multicolumn{7}{c}{ NT scan } \\
\hline
\end{tabular}


of tumor recurrence, data were analyzed until palliative situation was obvious, and scheduled surveillance according to the guidelines was stopped. Additionally, we compared the adherence to the Swiss surveillance guidelines between subgroups of patients who did or did not receive adjuvant chemotherapy.

The study was approved by the ethical committees of the participating centers, and was conducted in compliance with the institutional guidelines for experimental investigation with human subjects. All patients gave written informed consent before being enrolled into the study.

\section{Statistics}

Categorical variables were statistically analyzed with Fisher's exact test, and survival data with the log-rank test. Statistical analyses were performed using SPSS 13.0 for Macintosh (SPSS Inc., Chicago, IL). A $P$-value $<0.050$ was considered to be significant. All $P$-values were twosided.

\section{RESULTS}

\section{Patient Characteristics}

Datasets of all 129 eligible patients (75 male, 54 female) were available. Median age was 72.7 years (range 27.392.2 years), and median follow-up was 33.5 months (range 5.6-74.7 months). Initial tumor stages according to AJCC were stage I (pT1/pT2 pN0 cM0) in $23(17.8 \%)$, stage II (pT3/pT4 pN0 cM0) in 59 (45.7\%), and stage III (pTx pN1/ pN2 cM0) in 47 patients $(36.4 \%) .{ }^{23}$ Forty-four (34.1\%) patients received adjuvant chemotherapy. Indications for chemotherapy were stage III in 41 patients, locally advanced primary tumor (pT4) in two node-negative patients, and lymphatic vessel infiltration in one patient (pT3 pN0 G3 L1). Eighteen patients (14.0\%) recurred during follow-up, five of whom $(5 / 18,27.8 \%)$ underwent surgery again. Eleven patients $(8.5 \%)$ died during followup: six $(4.6 \%)$ for tumor-related causes and five $(3.9 \%)$ for reasons unrelated to the colon cancer and without evidence of recurrent disease. Three-year overall and disease-free survival was $94.7 \%$ (stage I $91.3 \%$, stage II $98.0 \%$, and stage III 92.3\%; $P=0.727$ ) and $83.5 \%$ (stage I $87.4 \%$, stage II $88.7 \%$, and stage III $76.2 \% ; P=0.101$ ), respectively. Three-year overall survival for patients with and without adjuvant chemotherapy was $95.1 \%$ and $94.5 \%$, respectively $(P=0.266)$. The corresponding three-year disease-free survival rates were $80.3 \%$ and $85.3 \%$, respectively $(P=0.114)$. All in- and outpatient records on laboratory results, imaging, and colonoscopy could be obtained. Return rate of the questionnaires was $82.2 \%$.

\section{Carcinoembryonic Antigen}

CEA in the context of surveillance had to be measured in 125 of 129 patients during follow-up; four patients had not reached the first time point for CEA measurement 6 months postoperatively. The median percentage of patients with surveillance according to the guidelines by CEA measurement was $32.8 \%(30.2-41.3 \%)$. Figure 1 shows a comparison of patients expected to undergo surveillance by CEA measurement with patients with surveillance according to the guidelines. In 20 of 125 patients $(16.0 \%)$ not a single CEA measurement was performed postoperatively.

Additionally, we compared adherence to the recommended CEA measurements between subgroups of patients who did or did not receive adjuvant chemotherapy. The median percentage of surveillance according to the guidelines by CEA measurements was $43.6 \%(41.8-53.6 \%)$ and $26.8 \%(20.8-32.7 \%)$ for patients who did and who did not receive chemotherapy, respectively $(P=0.064)$.

\section{Ultrasound and CT Scan}

US/CT in the context of surveillance had to be conducted in 118 of 129 patients during follow-up; 11 patients had not reached the first recommended time point for US/ CT. The median percentage of patients with surveillance according to the guidelines by US/CT was $31.7 \%$ (30.6$38.9 \%$ ). Figure 2 shows a comparison of patients expected to undergo surveillance by US/CT with patients with surveillance according to the guidelines. In 35 of 118 patients

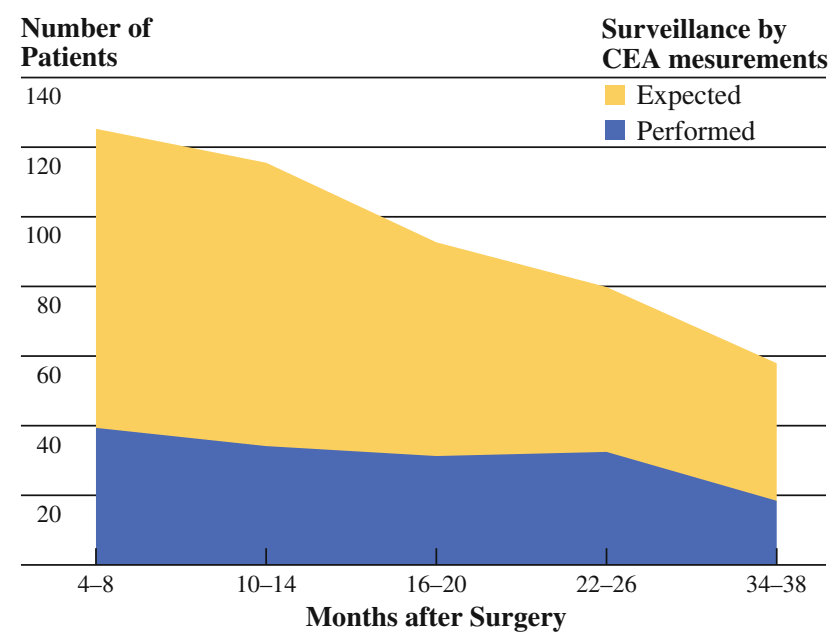

FIG. 1 Surveillance by CEA measurement. The number of patients expected to undergo surveillance based on Swiss guidelines is compared with the number of patients for whom surveillance actually was performed 


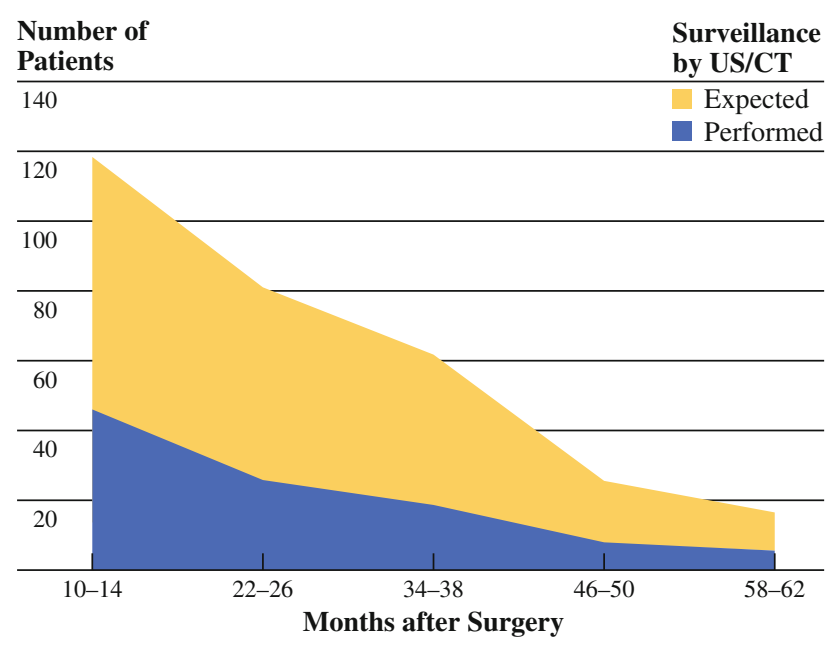

FIG. 2 Surveillance by ultrasound or CT scan. The number of patients expected to undergo surveillance based on Swiss guidelines is compared with the number of patients for whom surveillance actually was performed

(29.7\%) not a single ultrasound or CT scan was performed postoperatively. The median percentage of surveillance according to the guidelines by US/CT was $52.6 \%$ (41.6$66.6 \%$ ), and $21.4 \%(20.0-26.5 \%)$ for patients who did and who did not receive chemotherapy, respectively $(P=0.071)$.

\section{Colonoscopy}

A colonoscopy in the context of surveillance had to be conducted in 21 of 129 patients during follow-up; the remaining patients had not (yet) to be scheduled for surveillance colonoscopy. However, only five patients $(23.8 \%)$ received a colonoscopy within the defined timeframe. Moreover, preoperative colonoscopy was incomplete in 16 patients $(12.4 \%)$, five of whom $(5 / 16,31.3 \%)$ never got a completion colonoscopy postoperatively. Surveillance according to the guidelines by colonoscopy was performed in $40.0 \%$ ( 2 of 5 patients) and $18.8 \%$ ( 3 of 16 patients) for patients who did and who did not receive chemotherapy, respectively $(P=0.410)$.

\section{Overall Surveillance}

We also analyzed overall adherence to the surveillance guidelines, i.e., whether all CEA measurements, US/CT, and colonoscopies were performed according to the guidelines. Only 15 of 129 patients (11.6\%) underwent complete surveillance as recommended by the guidelines. On the other hand, $13.0 \%$ of patients did not have any of the recommended examinations after curative colon cancer resection.

\section{DISCUSSION}

We have analyzed the quality of surveillance after curative surgery for colon cancer among a cohort of Swiss patients. Adherence to national surveillance guidelines that mandate periodic measurement of CEA levels, abdominal US or CT scans, and colonoscopies was surprisingly poor. Less than one-third of patients received surveillance examinations according to the guidelines. Moreover, only $11.6 \%$ of patients underwent complete surveillance as recommended by the Swiss guidelines, while $13.0 \%$ of patient never had a single examination.

Data on the quality of surveillance after curative surgery for colon cancer are generally scarce. To our knowledge, this is the first investigation on adherence to a multipleitem surveillance program, i.e., including more than periodic surveillance colonoscopy. Other published studies exclusively report adherence to surveillance colonoscopy after surgery, as colonoscopy has been considered a key component of surveillance. ${ }^{24-28}$ In a recent Dutch study, Mulder and co-worker left the responsibility for organizing the surveillance colonoscopy - as in our study - to the general practitioners. ${ }^{24}$ Patients were not actively invited for surveillance. Only $30 \%$ of eligible patients underwent endoscopic surveillance within 1 year of the time point recommended in the national guidelines; $52 \%$ of patients had a delayed colonoscopy, and $18 \%$ had no colonoscopy at all. The authors concluded that an active follow-up invitation might be important. ${ }^{24}$ Data from the USA are similar: Cooper et al. identified 5,716 patients older than 65 years after curative surgery for colorectal cancer in 1991 from the Medicare-Surveillance Epidemiology and End Results database. ${ }^{25}$ Forty-nine percent of patients never underwent a colonoscopy during the first 3 years after surgery. Similarly, Elston Lafata and co-workers reported that only about two-thirds of colorectal cancer patients 30 years and older received the recommended colon examination during the initial year after surgery. ${ }^{26}$ Our findings are in line with the Dutch study ${ }^{24}$ However, we did not only analyze the use of colonoscopies, but the adherence to a multiple-item surveillance program, additionally including periodic CEA measurements and US/CT. The unexpectedly high proportion of patients $(13.0 \%)$ who never had a single surveillance examination in our study is very concerning. These patients were entirely excluded from the potential life-saving benefits of surveillance.

In the present investigation, the treating physicians were not questioned regarding why they did or did not adhere to the surveillance guidelines. Therefore, we can only speculate on potential reasons for not performing some or all surveillance examinations in the majority of patients. In some health care systems, access to some of the recommended examinations might be limited. However, a highly 
developed health care system-comparable to the USA as measured by the Organisation for Economic Co-operation and Development (OECD) health data-and mandatory health insurance coverage for all citizens grant access to surveillance for each and every Swiss patient. ${ }^{29-31}$ Waiting time for an ultrasound, a CT scan, or a colonoscopy is usually only a few days, and insurance companies cover the costs of all examinations recommended by the national surveillance guidelines. Another explanation might be that the treating physicians preferred one examination to another. However, adherence to the respective surveillance items (CEA, US/CT, and colonoscopy) consistently ranged around $30 \%$, indicating that this hypothesis is unlikely to hold. Third, physician age, i.e., the time that has elapsed since the doctor has trained, might explain differences in adherence to the guidelines as well. As we did not record physician age in this investigation, we cannot account for this effect. However, Johnson et al. conducted a survey of members of the Society of Surgical Oncology (SSO) and the American Society of Colon and Rectal Surgeons (ASCRS) to address this issue. ${ }^{32}$ Surveillance practice patterns did not vary substantially with practitioner age in this study, and the authors concluded that continuous postgraduate education is effective. ${ }^{32}$ Finally, it could be hypothesized that treating physicians were not aware of the need for surveillance per se and of the surveillance guidelines in detail, even though the SGG recommendations were published in a journal that is sent to every member of the Swiss Society of Physicians (FMH). This hypothesis is supported by a study from Israel in which general practitioners had to fill out a ten-item questionnaire on the proper timing of colonoscopy for surveillance and screening for colorectal cancer. ${ }^{33}$ The percentages of correct answer for the different items ranged from $6.2 \%$ to $58.5 \%$. The authors concluded that physicians' knowledge on surveillance and screening guidelines for colorectal cancer needed improvement. ${ }^{33}$

However, extensive knowledge on the details of the surveillance guidelines is less important than the general insight that surveillance after curative surgery for colon cancer is important. We believe that this is the number one reason why adherence to surveillance guidelines was so poor in our investigation. This is somewhat surprising, considering that all treating physicians in our study received a discharge letter where surveillance according to national guidelines was recommended for patients after curative colon cancer resection.

It is well documented that practice patterns for surveillance after curative surgery for colon or rectal cancer vary substantially, e.g., depending on whether surveillance is organized by a member of the SSO or the ASCRS. ${ }^{34,35}$ On the other hand, surveillance is not markedly intensified for higher Tumor-Node-Metastasis (TNM) stages as compared with early cancers. ${ }^{36}$ In our study, although far from optimal, the quality of surveillance was found to be considerably better in the subgroup of patients undergoing adjuvant chemotherapy as compared with patients who did not receive adjuvant treatment. It is possible that general practitioners considered these patients to be at higher risk for recurrence, and therefore performed surveillance examinations more thoroughly. Additionally, medical oncologists closely followed these patients for several months during the chemotherapy, and initiated surveillance. Therefore, our data support strongly the statement in the 2007 SGG recommendations that surveillance is an interdisciplinary process that has to be coordinated by a single person or institution. ${ }^{12}$

Additionally, it is critically important to convince health care professionals and patients of the potential life-saving benefits of surveillance. In this respect, educational efforts on the professional level as well as for lays are mandatory. As a first measure we have started to hand out a personalized surveillance schedule, including the dates when surveillance examinations should be scheduled, directly to our patients, giving them the opportunity to share the responsibility for surveillance with their treating physicians. However, as this change in practice pattern occurred only after closure of this trial, we do not yet have data on the effect of this measure. The creation of an (internetbased) recall system, as suggested by Mulder et al., might be another helpful tool. ${ }^{24}$

We would like to acknowledge the limitations of our study: first, it is a retrospective investigation. However, organizing and performing the surveillance examinations was deliberately left to the discretion of the respective treating physicians (general practitioners or medical oncologists). This study setting closely mimics the real situation in Switzerland, where most patient care after hospital discharge is performed by general practitioners. If, in the context of a prospective trial, they had been aware of the study objectives, they probably would have followed the guidelines more closely. Therefore, our results give a realistic view of surveillance performed after curative surgery for colon cancer in Switzerland. The setting of this trial (general practitioners referring their patients to an academic or university-affiliated hospital) and the fact that the patients were enrolled in a trial conceivably might have biased our data; however, this possible bias would be, if anything, in favor of better adherence to the surveillance guidelines. Another limiting factor of our study is the fact that the SGG guidelines were updated during the study period. ${ }^{20}$ It is well conceivable that surveillance of patients operated on before 2003 was not immediately adapted when the new guidelines came into effect. Third, the poor result could possibly be due in part to poor data capture, as we cannot track insurance data by a national identification 
number in Switzerland. However, all patients still alive were contacted by phone, and the return rate of the questionnaires was more than $80 \%$. Finally, our definition of surveillance according to the guidelines ( \pm 2 months for CEA and US/CT or \pm 4 months for colonoscopy of the recommended time point, respectively) might have influenced the results. However, e.g., of 483 CEA measurements, only $39(8 \%)$ in 21 patients were performed outside the defined time interval. We therefore conclude that our results have only marginally $(<10 \%)$ been affected by our definition.

\section{CONCLUSIONS}

The quality of surveillance after curative surgery for colon cancer is inadequate, even though access to surveillance is granted for everybody by the Swiss health care system. Further education of health professionals and patients regarding the potential life-saving benefits of surveillance is imperative. It is cardinal that quality of surveillance is critically analyzed in other countries with different health care systems as well.

DISCLOSURE The authors have no conflicts of interest to declare.

\section{REFERENCES}

1. Parkin DM, Bray F, Ferlay J, Pisani P. Global cancer statistics, 2002. CA Cancer J Clin. 2005; 55:74-108.

2. http://www.cancer.gov/cancertopics/types/colon-and-rectal. Accessed March 15, 2009.

3. Bohm B, Schwenk W, Hucke HP, Stock W. Does methodic longterm follow-up affect survival after curative resection of colorectal carcinoma? Dis Colon Rectum. 1993;36:280-6.

4. Prandi M, Lionetto R, Bini A, et al. Prognostic evaluation of stage B colon cancer patients is improved by an adequate lymphadenectomy: results of a secondary analysis of a large scale adjuvant trial. Ann Surg. 2002;235:458-63.

5. Kobayashi H, Mochizuki H, Sugihara K, et al. Characteristics of recurrence and surveillance tools after curative resection for colorectal cancer: a multicenter study. Surgery. 2007;141:67-75.

6. Ovaska JT, Jarvinen HJ, Mecklin JP. The value of a follow-up programme after radical surgery for colorectal carcinoma. Scand J Gastroenterol. 1989;24:416-22.

7. Renehan AG, Egger M, Saunders MP, O’Dwyer ST. Impact on survival of intensive follow up after curative resection for colorectal cancer: systematic review and meta-analysis of randomised trials. BMJ. 2002;324:813.

8. Jeffrey M, Hickey BE, Hider PN. Follow-up strategies for patients treated for non-metastatic colorectal cancer. Cochrane Database Syst Rev. 2007:CD002200.

9. Figueredo A, Rumble RB, Maroun J, et al. Follow-up of patients with curatively resected colorectal cancer: a practice guideline. BMC Cancer. 2003;3:26-0.

10. Grossmann EM, Johnson FE, Virgo KS, Longo WE, Fossati R. Follow-up of colorectal cancer patients after resection with curative intent-the GILDA trial. Surg Oncol. 2004;13:119-24.
11. Johnson FE, Virgo KS, Fossati R. Follow-up for patients with colorectal cancer after curative-intent primary treatment. $J$ Clin Oncol. 2004;22:1363-5.

12. Criblez D. Revidierte Konsensus-Empfehlungen zur Nachsorge nach koloskopischer Polypektomie und nach kurativ operiertem kolorektalem Karzinom. Schweiz Med Forum. 2007;7:994-99.

13. Desch CE, Benson AB, III, Somerfield MR, et al. Colorectal cancer surveillance: 2005 update of an American Society of Clinical Oncology practice guideline. J Clin Oncol. 2005;23: 8512-9.

14. Schmiegel W, Adler G, Fruhmorgen P, et al. Colorectal carcinoma: prevention and early detection in an asymptomatic population-prevention in patients at risk-endoscopic diagnosis, therapy and after-care of polyps and carcinomas. German Society of Digestive and Metabolic Diseases/Study Group for Gastrointestinal Oncology. Z Gastroenterol. 2000;38:49-75.

15. Abdalla EK, Vauthey JN, Ellis LM, et al. Recurrence and outcomes following hepatic resection, radiofrequency ablation, and combined resection/ablation for colorectal liver metastases. Ann Surg. 2004;239:818-25; discussion 25-7.

16. Cummings LC, Payes JD, Cooper GS. Survival after hepatic resection in metastatic colorectal cancer: a population-based study. Cancer. 2007;109:718-26.

17. Fernandez FG, Drebin JA, Linehan DC, Dehdashti F, Siegel BA, Strasberg SM. Five-year survival after resection of hepatic metastases from colorectal cancer in patients screened by positron emission tomography with F-18 fluorodeoxyglucose (FDGPET). Ann Surg. 2004;240:438-47; discussion 47-50.

18. Adam R, Delvart V, Pascal G, et al. Rescue surgery for unresectable colorectal liver metastases downstaged by chemotherapy: a model to predict long-term survival. Ann Surg. 2004;240:644-57; discussion 57-8.

19. Giacchetti S, Itzhaki M, Gruia G, et al. Long-term survival of patients with unresectable colorectal cancer liver metastases following infusional chemotherapy with 5-fluorouracil, leucovorin, oxaliplatin and surgery. Ann Oncol. 1999;10:663-9.

20. Criblez D. Die revidierten FAGAS-Empfehlungen zur Nachsorge nach koloskopischer Polypektomie und kurativ operiertem kolorektalem Karzinom. Schweiz Med Forum. 2004;4:611-17.

21. FAGAS. Nachsorge nach Resektion von kolorektalen Polypen und von kolorektalen Karzinomen. Schweizerische Ärztezeitung. 2001;82:1967-71.

22. Viehl CT, Guller U, Cecini R, et al. Upstaging due to sentinel lymph node procedure in colon cancer-a prospective multicenter study. Ann Surg Oncol. 2008;15:58-59.

23. Greene FL, Page DL, Fleming ID, Fritz AG, Balch CM, Haller DG, Morrow M. American Joint Committee Cancer Staging Manual. 6th ed. New York: Springer; 2002.

24. Mulder SA, Van Leerdam ME, Ouwendijk RJ, Bac DJ, Giard RW, Kuipers EJ. Attendance at surveillance endoscopy of patients with adenoma or colorectal cancer. Scand J Gastroenterol. 2007;42:66-71.

25. Cooper GS, Yuan Z, Chak A, Rimm AA. Patterns of endoscopic follow-up after surgery for nonmetastatic colorectal cancer. Gastrointest Endosc. 2000;52:33-8.

26. Elston Lafata J, Simpkins J, Schultz L, et al. Routine surveillance care after cancer treatment with curative intent. Med Care. 2005;43:592-9.

27. Harewood GC, Rathore O, Patchett S, Murray F. Assessment of adherence to published surveillance guidelines-opportunity to enhance efficiency of endoscopic practice. Ir Med J. 2008;101: 248-50.

28. Kahi CJ, Rex DK, Imperiale TF. Screening, surveillance, and primary prevention for colorectal cancer: a review of the recent literature. Gastroenterology. 2008;135:380-99. 
29. http://www.ecosante.org/index2.php?base=OCDE\&langh=ENG\& langs=ENG. Accessed March 15, 2009.

30. http://www.bag.admin.ch/themen/krankenversicherung/00300/ index.html?lang=en. Accessed March 15, 2009.

31. Herzlinger RE, Parsa-Parsi R. Consumer-driven health care: lessons from Switzerland. JAMA. 2004;292:1213-20.

32. Johnson FE, Novell LA, Coplin MA, Longo WE, Vernava AM, Wade TP, et al. How practice patterns in colon cancer patient follow-up are affected by surgeon age. Surg Oncol. 1996;5:12731.

33. Zbidi I, Hazazi R, Niv Y, Birkenfeld S. Colonosopy screening and surveillance of colorectal cancer and polyps: physicians' knowledge. Isr Med Assoc J. 2007;9:862-5.
34. Virgo KS, Wade TP, Longo WE, Coplin MA, Vernava AM, Johnson FE. Surveillance after curative colon cancer resection: practice patterns of surgical subspecialists. Ann Surg Oncol. 1995;2:472-82.

35. Johnson FE, Longo WE, Ode K, et al. Patient surveillance after curative-intent surgery for rectal cancer. Int J Oncol. 2005;27: 815-22.

36. Vernava AM III, Longo WE, Virgo KS, Coplin MA, Wade TP, Johnson FE. Current follow-up strategies after resection of colon cancer. Results of a survey of members of the American Society of Colon and Rectal Surgeons. Dis Colon Rectum. 1994;37:57383. 